\title{
Correction to: Security of Public Key Encryption Against Resetting Attacks
}

Juliane Krämer and Patrick Struck

\section{Correction to:}

Chapter "Security of Public Key Encryption Against Resetting Attacks" in: K. Bhargavan et al. (Eds.): Progress in Cryptology

- INDOCRYPT 2020, LNCS 12578, https://doi.org/10.1007/978-3-030-65277-7_23

In the original version of this book there are several formatting mistakes. This has been corrected. 